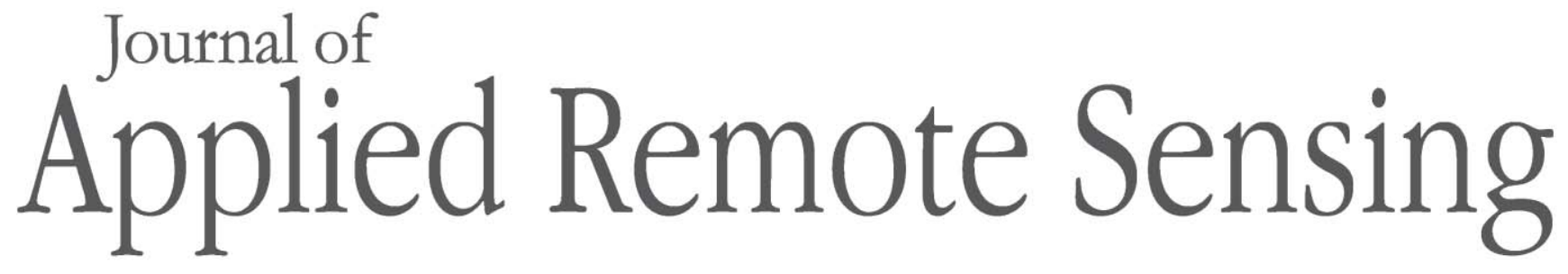

\title{
Characterization of the Sonoran desert as a radiometric calibration target for Earth observing sensors
}

Amit Angal

Gyanesh Chander

Xiaoxiong Xiong

Taeyoung Choi

Aisheng $\mathrm{Wu}$ 


\title{
Characterization of the Sonoran desert as a radiometric calibration target for Earth observing sensors
}

\author{
Amit Angal, ${ }^{a}$ Gyanesh Chander, ${ }^{\mathrm{b}}$ Xiaoxiong Xiong, \\ Taeyoung Choi, ${ }^{d}$ and Aisheng $W^{d}$ \\ ${ }^{a}$ Science Systems and Applications, Inc., 10210 Greenbelt Road, Lanham, Maryland 20706 \\ amit.angal@ssaihq.com \\ ${ }^{\mathrm{b}}$ SGT, Inc., United States Geological Survey Earth Resources Observation and Science \\ Center, Sioux Falls, South Dakota 57198 \\ ${ }^{\mathrm{c}}$ NASA Goddard Space Flight Center, Sciences and Exploration Directorate, Greenbelt, \\ Maryland 20771 \\ d Sigma Space Corporation, Lanham, Maryland 20706
}

\begin{abstract}
To provide highly accurate quantitative measurements of the Earth's surface, a comprehensive calibration and validation of the satellite sensors is required. The NASA Moderate Resolution Imaging Spectroradiometer (MODIS) Characterization Support Team, in collaboration with United States Geological Survey, Earth Resources Observation and Science Center, has previously demonstrated the use of African desert sites to monitor the long-term calibration stability of Terra MODIS and Landsat 7 (L7) Enhanced Thematic Mapper plus (ETM+). The current study focuses on evaluating the suitability of the Sonoran Desert test site for post-launch long-term radiometric calibration as well as cross-calibration purposes. Due to the lack of historical and on-going in situ ground measurements, the Sonoran Desert is not usually used for absolute calibration. An in-depth evaluation (spatial, temporal, and spectral stability) of this site using well calibrated L7 ETM+ measurements and local climatology data has been performed. The Sonoran Desert site produced spatial variability of about 3 to 5\% in the reflective solar regions, and the temporal variations of the site after correction for view-geometry impacts were generally around $3 \%$. The results demonstrate that, barring the impacts due to occasional precipitation, the Sonoran Desert site can be effectively used for cross-calibration and longterm stability monitoring of satellite sensors, thus, providing a good test site in the western hemisphere. ( 2011 Society of Photo-Optical Instrumentation Engineers (SPIE). [DOI: 10.1117/1.3613963]
\end{abstract}

Keywords: Sonoran; calibration; bidirectional reflectance distribution function; ETM+, Libya4, Hyperion; characterization; moderate resolution imaging spectroradiometer.

Paper 11022LR received Feb. 9, 2011; revised manuscript received May 11, 2011; accepted for publication Jun. 28, 2011; published online Jul. 22, 2011.

\section{Introduction}

Use of stable ground targets for sensor cross-calibration and long-term stability monitoring requires an in-depth characterization and understanding of the targets. Since these ground targets are believed to exhibit minimal changes with time, the need for acquiring near simultaneous ground measurements can be relaxed to a certain extent. One such pseudo-invariant site, is the Sonoran Desert located near the United States and Mexico border. It is referenced in the Worldwide Reference System 2 with path 38 and row 38. ${ }^{1}$ Previous work focused on demonstrating the use of this site for long-term stability monitoring of satellite sensors, such as the Along Track Scanning Radiometer-2, Terra Moderate Resolution Imaging Spectroradiometer (MODIS), and Landsat 7 (L7) Enhanced Thematic Mapper Plus (ETM+) ${ }^{2,3}$ Intraseasonal fluctuations associated with the Mexican monsoon systems are well documented in the case of

$1931-3195 / 2011 / \$ 25.00$ @ 2011 SPIE 


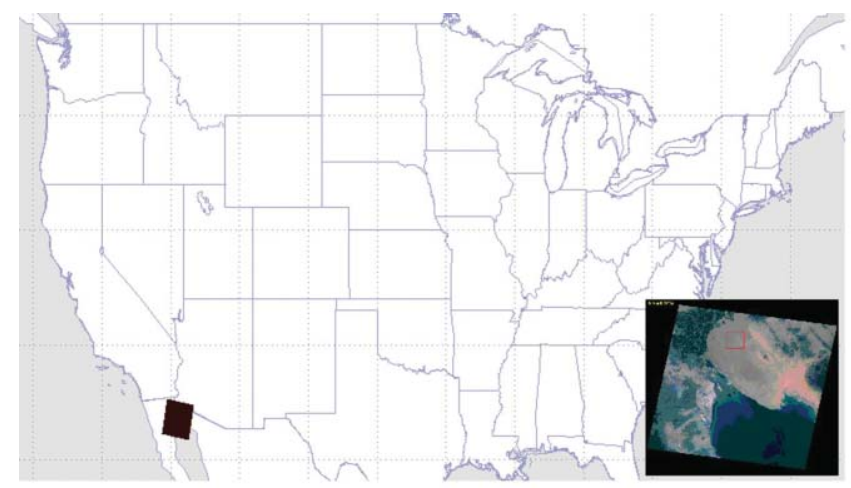

Fig. 1 Geographical location of the Sonoran Desert with a L7 ETM+ acquisition from April, 2003.

the semi-arid Sonoran Desert. ${ }^{4}$ The following article evaluates the impacts of such fluctuations on the site characteristics and their effect on the retrieved top-of-atmosphere (TOA) reflectance. This study uses well-calibrated high spatial resolution L7 ETM+ measurements to evaluate the spatial uniformity and stability. In addition, hyperspectral observations from Earth Observing-1 (EO-1) Hyperion are used to study the spectral characteristics of the Sonoran Desert site. The TOA reflectance trends of ETM + since its launch in April 1999 have been extremely stable with changes less than $0.4 \%$ per year in its TOA reflectance over pseudo-invariant ground sites ${ }^{5,6}$ and, hence, are used to evaluate the temporal stability of the site.

\section{Site and Sensor Description}

\subsection{Test Site Description}

The Sonoran Desert in Mexico $\left(+32.25^{\circ},-114.65^{\circ}\right)$ is a large flat semi-arid region with limited vegetation cover and known seasonal variations such as a variable water table. The average altitude of the site is $0.2 \mathrm{~km}$ above sea level, and it has horizontal visibilities of 30 to $45 \mathrm{~km} .{ }^{3}$ Due to its specific geographical location, the Sonoran Desert occasionally encounters unpredictable rainfall in both the winter and summer months. The summer rainfall season typically extends from June through September, and the mean temperature in the region typically extends from about $50^{\circ} \mathrm{F}$ in the winter months up to $100^{\circ} \mathrm{F}$ in the late summer months. ${ }^{7}$ Figure 1 shows the geographical location of the Sonoran Desert along with a composite image of the region acquired by L7 ETM+ bands 3, 2, 1 from April, 2003. Fundamentally, the region of interest (ROI) should be located in a spatially uniform area large enough to accommodate the sampling of a large number of pixels and to minimize atmospheric adjacency effects due to light scattered from outside the target region. Considering these criteria, a $15 \times 15 \mathrm{~km}$ ROI centered around $\left(+32.25^{\circ},-114.65^{\circ}\right)$ was selected and this fixed ROI was used to evaluate the various site characteristics. ${ }^{1}$

\subsection{Sensor Description}

The high spatial resolution (30 m) multispectral bands of L7 ETM+ have been acquiring high quality data since its launch on April 15, 1999, and have significantly contributed to the 36+ year time series of land observations of the Landsat Program. Due to its higher spatial resolution and long-term stable performance, the L7 ETM+ can be effectively used to evaluate the spatial characteristics of a test site. A 5\% uncertainty requirement is specified for the reflective solar bands (RSB) at-sensor spectral radiance calibration. ${ }^{8}$ Because the repeat cycle of L7 ETM+ is 16 days, a near-nadir acquisition of the Sonoran Desert test site can be obtained once every 16 days. Due to different sensor and solar view geometry, the surface and atmospheric bidirectional 
reflectance distribution function (BRDF) may impact the retrieved TOA reflectances. To account for these uncertainties and to reliably evaluate the temporal stability of the site, a semi-empirical BRDF (Roujean) model has been adopted to mitigate the impacts due to the view geometry differences on the retrieved TOA reflectances. ${ }^{9}$ EO-1 Hyperion is a high spatial resolution $(30 \mathrm{~m})$ grating imaging spectrometer capable of providing near-continuous information regarding the spectral signature of the target from 0.4 to $2.5 \mu \mathrm{m}$. Hyperion has 220 spectral bands with a spectral resolution of $10 \mathrm{~nm}$, and the swath of the acquisition is typically about 7.5 $\mathrm{km}$. As part of the A.M. constellation, the L7 ETM+ and EO-1 Hyperion were used to acquire data near-simultaneously with only a minute difference in their acquisition times; however, due to the subsequent lowering of the EO-1 orbit, this might not be the case for the data acquired in the late part of the EO-1 mission. Hyperion is radiometrically stable to within $5 \%$, based on the lunar calibration observations in comparison with the United States Geological Survey Robotic Lunar Observatory (ROLO) model. ${ }^{6}$

\section{Site Evaluation}

\subsection{Spatial Uniformity}

Spatial uniformity is especially important in sensor cross-calibration because registration errors introduce significant uncertainties when the surface is not uniform. Using the baseline ROI defined in Sec. 2.1, the image statistics were computed to obtain the mean and standard deviation of the ROI on a band-by-band basis. The coefficient of variation (CV) metric, as defined in Eq. (1), is a ratio of standard deviation to the mean TOA reflectance for a given window of pixels. The smaller the value of CV, the better is the spatial uniformity of the selected ROI. Using a $15 \times 15 \mathrm{~km}$ moving window around the Sonoran Desert region, the ROI with the least value of $\mathrm{CV}$ was selected for this study

$$
C V(\lambda)=|\sigma(\lambda, t) / \operatorname{refl}(\lambda, t)|^{*} 100 .
$$

To track the spatial uniformity of the site over time, all available ETM+ images (247) over the chosen ROI were processed. The images with the presence of clouds were excluded using the brightness temperature thresholds and 221 scenes were retained for further computations. ${ }^{10}$ Also, the pixels impacted by ETM+ scan line corrector failure were excluded from the calculations. Using the mean and standard deviation computed for every ROI, a wavelength dependent CV was calculated. Table 1 summarizes the mean TOA reflectance and the $\mathrm{CV}$ metric derived for all of the ETM+ bands using the Sonoran Desert ROI. The time-averaged spatial uniformity metric generally ranges around 3 to $5 \%$ depending on the selected wavelength. However, the time variability of this metric exhibit changes more with the difference between the maximum and minimum value of the metric ranging from 2 to $8 \%$. The possible reasons for this might obviously be nonhomogeneity of the selected ROI, but variations in the atmospheric and surface reflectance conditions could also play a role. In the case of the Libya-4 pseudo-invariant site in the North African desert, this CV metric is generally seen to be within 3\%.

Table 1 Spatial uniformity metric for the Sonoran Desert site using multi-year ETM+ observations.

\begin{tabular}{lllllll}
\hline \hline Wavelength & $0.48(\mu \mathrm{m})$ & $0.56(\mu \mathrm{m})$ & $0.66(\mu \mathrm{m})$ & $0.83(\mu \mathrm{m})$ & $1.64(\mu \mathrm{m})$ & $2.2(\mu \mathrm{m})$ \\
mean refl & 0.20 & 0.24 & 0.32 & 0.37 & 0.42 & 0.36 \\
CV Metric & $4.52 \%$ & $4.35 \%$ & $3.73 \%$ & $3.36 \%$ & $3.05 \%$ & $3.64 \%$ \\
Min. & $2.91 \%$ & $2.82 \%$ & $2.70 \%$ & $2.26 \%$ & $2.24 \%$ & $3.14 \%$ \\
Max. & $7.33 \%$ & $6.17 \%$ & $4.59 \%$ & $4.1 \%$ & $3.46 \%$ & $4.26 \%$ \\
\hline \hline
\end{tabular}




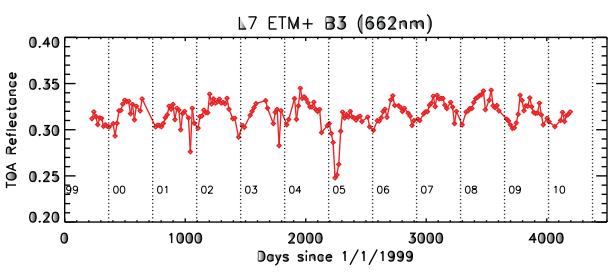

(a)

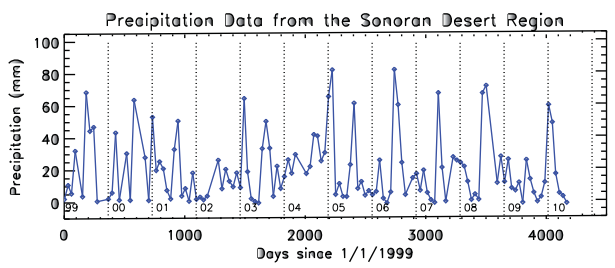

(b)

Fig. 2 L7 ETM+ band 3 TOA reflectance trending and precipitation measurements from the Sonoran Desert region.

\subsection{Temporal Stability}

Since its first acquisition in 1999, L7 ETM+ has been acquiring a near-nadir image of the Sonoran Desert region once every 16 days. About 247 near-nadir images from launch to June, 2010 were used to evaluate the temporal stability of the site. Using the fixed ROI discussed earlier, the TOA reflectances were calculated for all multispectral bands of L7 ETM+. ${ }^{5}$ For example, an average TOA reflectance over the selected ROI is tracked for ETM+ band 3 $(662 \mathrm{~nm})$ as a function of time and can be seen in Fig. 2(a). In general, the TOA reflectance is stable, except for the seasonal oscillations predominantly due to different view geometries during acquisition. In addition, certain time periods (early months of 2005) show lower reflectance values compared to the general trending. TOA reflectance trending ETM+ band 3 from other Committee on Earth Observation Satellites (CEOS) standard reference pseudo-invariant sites does not exhibit such a trend, which indicates that the change in surface or atmospheric conditions might have an impact on the retrieved TOA reflectance. Also, TOA reflectance trending from other sensors such as Terra and Aqua MODIS and L5 TM also exhibited similar trends across all wavelengths. ${ }^{2}$

To further understand these trends, precipitation measurements over the Sonoran Desert region were obtained from the Global Historic Climatology Network (GHCN). ${ }^{11}$ The GHCN database consists of monthly surface observations from $\sim 7000$ stations worldwide. Figure 2(b) shows the monthly averaged precipitation measurements plotted for the entire timeline of the L7 ETM+ operation. In general, most precipitation occurs during July through September, and the months of March through June seem to indicate the least precipitation. The early months of the year periodically indicate significant amounts of precipitation, especially in January and February 2005. A combination of low solar elevation angle during the winter months and higher precipitation during this year explains the lower values of the retrieved TOA reflectances in the early months of the year 2005 as seen in Fig. 2.

Using pseudo-invariant sites, the long-term TOA reflectance change for L7 ETM+ is within its calibration uncertainty specification of $\pm 5 \%{ }^{5}$ Hence, the temporal trends of the TOA reflectance will provide us with a reasonable estimate of the site's stability over the 10-year time period beginning February 2000. To mitigate the impacts due to surface and atmospheric BRDF, a semi-empirical BRDF model has been adopted using ETM+ TOA reflectance measurements made during the time period from January 2003 to December 2004. The kernel driven BRDF model $^{9}$ was used to compute the BRDF normalized reflectances. The measured TOA reflectances for all of the ETM+ bands were normalized by the BRDF modeled reflectances and fitted to a simple linear model. The basic assumption in using this approach to evaluate the temporal

Table 2 Temporal stability metric for the Sonoran Desert site.

\begin{tabular}{|c|c|c|c|c|c|c|}
\hline Wavelength & $0.48(\mu \mathrm{m})$ & $0.56(\mu \mathrm{m})$ & $0.66(\mu \mathrm{m})$ & $0.83(\mu \mathrm{m})$ & $1.64(\mu \mathrm{m})$ & $2.2(\mu \mathrm{m})$ \\
\hline All Data & $3.36 \%$ & $3.37 \%$ & $3.58 \%$ & $3.51 \%$ & $3.67 \%$ & $5.97 \%$ \\
\hline 2005 exclude & $2.95 \%$ & $2.55 \%$ & $2.53 \%$ & $3.16 \%$ & $2.79 \%$ & $5.13 \%$ \\
\hline
\end{tabular}




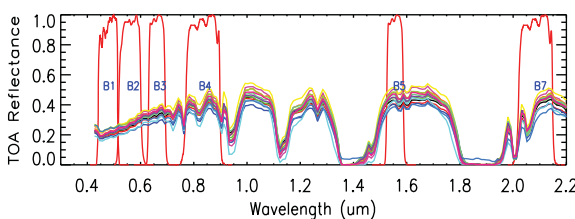

(a)

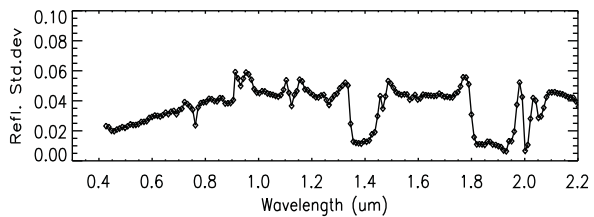

(b)

Fig. 3 Spectral signature of the Sonoran Desert target as retrieved using EO-1 Hyperion.

stability is that the long-term drift in the TOA reflectance of the sensor is within its calibration uncertainty specification. Table 2 summarizes the standard deviation of the TOA reflectance trends after BRDF normalization. Similar to the spatial uniformity metric, the temporal stability metric can also be defined based on standard deviation. The temporal standard deviation should be calculated after BRDF compensation with lower values indicating minimal change in site characteristics. In general, the long-term temporal stability metric values are around 3\%, except the $2.2 \mu \mathrm{m}$ band (ETM+ band 7), which shows variations of about $5 \%$. The temporal stability of $5.97 \%$ in the $2.2 \mu \mathrm{m}$ band indicates a larger variability of the site due to changes in the surface and atmospheric conditions. The temporal stability metric was recalculated after excluding the measurements in the early months of 2005 , which were impacted by precipitation. As expected, better temporal stability values were obtained, indicating that site variability is generally within $3 \%$, except for the longer wavelength band. There might also be some contributions due to the inadequacy of the BRDF model in accounting for these changes. Using the same methodology, the temporal stability for the Libya 4 test site was seen to be less than $2 \%$ at all wavelengths.

\subsection{Spectral Stability}

Only a limited number of cloud-free EO-1 Hyperion images over the Sonoran Desert region are available for evaluation of the spectral signature. The changes in the spectral signature could reflect the actual site changes in addition to the impacts due to atmospheric profile changes. Figure 3(a) shows the TOA reflectance profile obtained from the eleven cloud-free EO-1 Hyperion images acquired over the Sonoran Desert region along with the ETM+ Relative Spectral Response (RSR) for all RSB. The TOA reflectance profile is obtained using the EO-1 Hyperion level $1 \mathrm{G}$ product by choosing a $3 \times 3 \mathrm{~km}$ uniform ROI in each of these images. Due to the limited swath of the EO-1 Hyperion combined with a lowered orbit, the Sonoran desert scenes are acquired with different ROI. The standard deviation computed using all eleven images is shown in Fig. 3(b). The deviation in the spectral signature can be attributed to the variations in the surface and atmospheric conditions of the Sonoran Desert region. In general, variations in the Visible and Near-IR (VNIR) bands are between 3 to $4 \%$ as compared to about $5 \%$ seen in the Short-Wave Infrared (SWIR) channels.

Variations in atmospheric composition over a selected target complicate the ability of a remote sensor to assess the (spectral) stability of a ground site. The absorption features of atmospheric gases such as $\mathrm{H}_{2} \mathrm{O}, \mathrm{O}_{2}$, and $\mathrm{CO}_{2}$ are evident in Fig. 3(a). Concentrations of atmospheric gases vary on diurnal, seasonal, and decadal scales and can introduce uncertainties in the retrieved TOA reflectance. The bandpass RSR for ETM+ bands 1 to 5 and 7 are overlaid on the Hyperion spectra. It can be seen that bands 4 and 7 in particular contain distinct absorption features that can impact the retrieved reflectance.

\section{Summary}

Characterization of a site is the primary step for the development of a robust methodology to perform calibration and validation of satellite sensors using stable ground targets. This study focused on using the TOA measurements from the L7 ETM+ and EO-1 Hyperion sensor over the 
Sonoran Desert site to evaluate the following parameters: spatial uniformity, temporal stability, and spectral variability. The large L7 ETM+ dataset enables us to more accurately characterize the site. In general, the spatial uniformity metric shows that the site is generally stable, within 3 to $5 \%$, with more variations in the shorter wavelength bands. The temporal stability due to the combined impacts of surface and atmospheric changes over a span of 10 years is generally around 3\%, except for the $2.2 \mu \mathrm{m}$ bands, where it is around $5.97 \%$. The impacts of seasonal precipitation can have a significant effect on the long-term TOA reflectance trending, especially in the winter months. The variations in the spectral signature of the site were tracked using the limited EO-1 Hyperion scenes. The VNIR channels exhibit smaller variations (4\%) as compared to the variations seen in the SWIR bands (5\%). This study demonstrates that the Sonoran Desert site can be effectively used for long-term monitoring and cross-calibration of satellite sensors. The various metrics developed in this work will be applied to all of the CEOS pseudo-invariant test sites for a better understanding of the various characteristics exhibited by these sites.

\section{Acknowledgments}

The authors thank Thomas Adamson (SGT) and Brian Wenny (MCST) for providing helpful comments in the technical review of this manuscript. Work at SGT, Inc. performed under U.S. Geological Survey contract G10PC00044.

\section{References}

1. G. Chander, J. B. Christopherson, G. L. Stensaas, and P. M. Teillet, "Online catalogue of world-wide test sites for the post-launch characterization and calibration of optical sensors," in Proceedings of the International Astronautical Federation, 3, 2043-2051 (2007).

2. A. Angal, X. Xiong, T. Choi, G. Chander, and A. Wu, "Using the Sonoran and Libyan Desert Test Sites to monitor the temporal stability of the reflective solar bands for Landsat 7 ETM+ and Terra MODIS sensors," J. Appl. Rem. Sens. 4, 043525 (2010).

3. D. L. Smith, C. T. Mutlow, and C. R. Nagaraja Rao, "Calibration monitoring of the visible and near-infrared channels of the along-track scanning radiometer- 2 by use of stable terrestrial sites," J. Appl. Opt. 41(3), 515-523 (2002).

4. S. Mullen, J. Schmitz, and N. Rennó, "Intraseasonal variability of the summer monsoon over southeast Arizona," Mon. Weather Rev. 126, 3016-3035 (1998).

5. G. Chander, X. Xiong, T. Choi, and A. Angal, "Monitoring on-orbit calibration stability of the Terra MODIS and Landsat 7 ETM+ sensors using pseudo-invariant test sites," Remote Sens. Environ. 114, 925-939 (2010).

6. S. Ungar, E. Middleton, L. Ong, and P. Campbell, "EO-1 Hyperion Onboard Performance over Eight years : Hyperion Calibration," in Proceedings of 6th EARSeL, Imaging Spectroscopy SIG Workshop, March (2009).

7. R. Houk, "Sonoran Desert," Southwest Parks and Monuments Association, pp. 6-16, (2000).

8. J. Barsi, B. Markham, D. Helder, and G. Chander, "Radiometric calibration status of Landsat 7 and Landsat 5," Proc. SPIE 6744, 67441F (2007).

9. J. L. Roujean, M. J. Leroy, and P. Y. Deschamps, "A bidirectional reflectance model of the earth's surface for the correction of remote sensing data," J. Geophys. Res. 97, 20455-20468 (1992).

10. A. Wu and Q. Zhong, "A method for determining the sensor degradation rates of NOAA AVHRR channels 1 and 2," J. Appl. Meteorol. 33, 118-121 (1994).

11. T. Peterson and V. Russell, "An overview of the global historical climatology network temperature database," Bull. Am. Meteorol. Soc. 78, 2837-2849 (1997).

Biographies and photographs of the authors not available. 